\title{
A BIBLIOGRAPHIC REVIEW OF WIDEBAND DTV NETWORKS USING TRANSMISSION DIVERSITY TECHNIQUES
}

\section{Mauricio Vilela Guerra}

Doctorate Degree in Engineering from the Pontifical Catholic University of Rio de Janeiro (PUC/Rio)

Rio de Janeiro, RJ, Brazil

mauricio.guerra@cefet-rj.br

\section{Marcelo de Jesus Rodrigues da Nóbrega}

Post-Doctorate Degree in Engineering from the State University of Rio de Janeiro (UERJ)

Rio de Janeiro, RJ, Brazil. engmarcelocefet@terra.com.br

\begin{abstract}
This paper presents results on Delay Spread giving a first complete bibliographic review applied to Digital Terrestrial Television (DTV) using distributed transmission schemes. A survey of the status of current research development is achieved from a possible trend recommended by a measurement campaign carried out in a suburban single frequency network (SFN) with two synchronized transmitters. Discussion of possible issues regarding reception conditions are considered and the constant need to fulfill modern technical requirements like spectral efficiency and system flexibility are also addressed. Theoretical, computer simulation, experimental, and field trial results are presented in this article confirming the improvements associated with source diversity networks.
\end{abstract}

Keywords: Single Frequency Networks, Digital Television, Spectral Efficiency. 


\section{RESUMO}

\section{UMA REVISÃO BIBLIOGRÁFICA DE REDES DE TV DIGITAL BANDA LARGA UTILIZANDO TÉCNICAS DE DIVERSIDADE DE TRANSMISSÃO}

Este artigo apresenta resultados do espalhamento de retardo, fornecendo em primeira mão uma revisão bibliográfica completa aplicada à Televisão Digital Terrestre utilizada em esquemas de transmissão distribuída. Um levantamento da situação atual do desenvolvimento da pesquisa é obtido a partir de uma possível tendência recomendada por uma campanha de medição realizada em uma rede de frequência única urbana com dois transmissores sincronizados. A discussão de possíveis questões relacionadas às condições de recepção é considerada e a constante necessidade de atender aos requisitos técnicos modernos, como eficiência espectral e flexibilidade do sistema, também é abordada. Resultados teóricos, de simulação computacional, experimentais e de ensaios de campo são apresentados neste artigo, confirmando as melhorias associadas às redes de diversidade de fontes.

Palavras-chave: Redes de Frequência Única. Televisão Digital. Eficiência Espectral.

\section{INTRODUCTION}

The predicted analogue switch-off in Brazil and the expected demand for DTV services such as High Definition Television (HDTV) has already become part of the modern technical requirement. Nevertheless, limited by frequency capacity, the terrestrial television platforms require more efficient transmission systems to fulfill the demands of market and allow launch of new services.

As presented in the measurement campaign carried out in a suburban SFN network with two synchronized transmitters (Guerra, 2012), considering mobile applications of the Brazilian DTV standard, the mode 1 guard interval provides a suitable guard interval length of $15.75 \mu \mathrm{s}$. Given that for the system the decrease of the guard interval is one of the ways to obtain an additional gain of information in number of bits, the measured data proved an extra transmission capacity of $4.04 \mu \mathrm{s}$, which can be translated in terms 
of a decrease for the required minimum guard interval length and the potential increase of spectral efficiency in the system (Takada, 2006).

Similarly to the digital broadcasting system adopted in Brazil, Digital Video Broadcasting standard (DVB-T) also uses Coded Orthogonal Frequency Division Multiplex (COFDM) modulation and coding schemes where new techniques have been introduced so that allowing spectral efficiency improvement (ETSI EN 300744 (V1.6.1), 2008). Notice the Terrestrial Integrated System Digital Broadcasting (ISDB-T) which is the Brazilian Digital Television (DTV) standard developed by Japan in May 1999 can be deemed as a derivative of DVB-T because of their similar technical features and system parameters. However, compared with DVB-T, ISDB-T has two major improvements. First, the interleaver with longer depth is used to improve the mobile reception performance. Second, the key technology called bandwidth segmented transmission OFDM (BST-OFDM) enables ISDB-T the capability of supporting multiple services.

Induced by the trend recommended in (Guerra, 2012), this article provides an overview of the current research development regarding spectral efficiency, system flexibilities and reception conditions associated with distributed transmission schemes.

\section{DEVELOPMENT}

\subsection{Survey of Current Research Development Status}

Currently, many researchers are exploring the spectral efficiency of terrestrial television platforms in order to meet customers' interest for quality video content and High definition TV (HDTV). The second generation terrestrial DVB-T2 standard studied in (Polak, 2015) presents one of the main innovations in terms of system configurations that is the possibility of applying advanced multiple-input single-output (MISO) transmission technique. Known as the MISO processing option, it is possible to use two transmitting antennas which, however, do not radiate the same broadcasted signal as at single-input single-output (SISO) mode (Polak, 2012),(I. Eizmendi, 2011),( L. Eizmendi and et al.,2013). As a consequence, this new transmission mode reduces the destructive spectral interferences caused by the presence of similar-strength signals normally seen in a "standard" Single Frequency Network (SFN) (Guerra, 2012),(Polak, 2015),(TS 
102831 V1.2.1, 2012). In fact, this is translated in terms of lower bit error rate (BER) values for all carrier-to-noise (C/N) ratios (Rohde \& Schwarz, 2012) when compared to the SISO method as explored in (Guerra, 2012). In (Polak, 2015), for broadcasting the DVB-T2 content, the SISO and MISO technique can be used and transmitters also include a channel simulator module. Two DVB-T2 transmitters were used to transmit DVB-T2 HDTV services using the new modulation and coding technique introduced by the DVBT2 standard. In other words, the possibility of using the 256-QAM mode allows a higher number of bits to be carried per data cell, which automatically increases the spectral efficiency of the system considering the same fixed reception scenario as occurred in (Guerra, 2012) and a rooftop outdoor antenna. Notice the DVB-T2 standard also support $16 \mathrm{k}$ and $32 \mathrm{k}$ transmission modes that allows the increase of the guard interval length without decreasing the spectral efficiency of the system (M. Takada, 2006),(DVB Project Office,2012). Three different transmission scenarios were evaluated in (Polak, 2015) but only the first two were regarded in this paper. In the first one, the MISO method was tested and the TV signals for both transmitters are broadcasted through an additive white Gaussian noise (AWGN) reference channel. Afterwards the measurement was repeated, but this time the TV signal from the master (TX1) and slave (TX2) transmitter was broadcasted through a Rayleigh channel with twenty independent paths (TS 102 831 V1.2.1, 2012),( Fisher, 2010) and the reference Gaussian channel, respectively. Being perhaps the most important result, the well-known negative impact of the Rayleigh channel (Morgade, 2013),(Morgade, 2014) on the broadcasted HDTV services turned out to be highly attenuated since there was only a small increase of BER ratios in the channel when compared with AWGN reference scenario. In MISO mode the same experiments were repeated using a Ricean channel (Guerra, 2012) and the obtained results were comparable with results obtained with the Rayleigh channel. On the other hand, the second scenario which was very similar to the first one the SISO mode was tested using two AWGN channel models and it fails. As expected, at the receiver, all participating sites that transmit the signal synchronously (frequency, content and time), results in an overlay of more-or-less identical signals which can lead to significant destructive spectral interference and hence higher BER values for all considered $\mathrm{C} / \mathrm{N}$ ratios (Rohde \& Schwarz, 2012). The results were slightly better when the HDTV content from TX1 and TX2 was transmitted through Rayleigh and Gaussian channels. The constructive echoes from the Rayleigh channel helped to decrease BER ratios before channel decoding. 
From 2012 onwards, many researchers deal with exploring DVB-T2 performances, when MISO mode is applied. Although it has been fundamentally designed for fixed reception, the DVB-T2 standard is also feasible in portable and mobile devices if appropriate set of parameters is used. Perhaps the main benefit of the DVB-T2 is the possibility to increase the capacity in digital terrestrial television (DTT) and this was earlier evaluated through the results obtained in (Guerra, 2012). Actually, the DVB-T2 provides a minimum increase in capacity of at least $30 \%$ in comparison to the original DVB-T standard in equivalent reception condition using existing receiving equipment (DVB BlueBook A133, 2009). Another important novelty in the DVB-T2 are low-density parity-check (LDPC) codes combined with Bose-Chaudhuri-Hocquenghem $(\mathrm{BCH})$, used as protection against interference and noise (Richardson, 2003). They offer excellent performance resulting in a very robust signal reception in various signal transmission condition. In (Tralic, 2012) a simulation and measurement of digital television signal transmission according to the DVB-T2 standard in Single-Frequency Networks (SFN) over few models of the fading channels was presented. The reception of the DVB-T2 signals is evaluated considering eight transmission conditions basically described over three different types of fading channels (Gaussian, Ricean, and Rayleigh). Among the fading channel models were Gaussian (AWGN), Ricean's DVBT-F - which simulates fixed reception, Rayleigh's DVBT-P - that simulates the portable reception, the TU06 and TU12 channel models - that simulate mobile reception conditions, and DTG long and DTG medium channels (having Ricean distribution) and DTG short channel (having Rayleigh distribution). Simulation results were obtained using the DVB-T2 test which performed dichotomic search for SNR (Signal to Noise Ratio) at target BER of $10^{-7}$ after LDPC decoder (DVB Document A122, 2008). In addition, current receiver channel estimator uses only ideal channel estimation, which means that the channel response is obtained from the channel itself and not from the pilot signals as it was done in the measurements that performs real channel estimation, thus increasing required SNR ( $\approx 10 \mathrm{~dB})$. For static multipath channels, frequency response is calculated directly from channel parameters. On the other side, for the mobile channels with Doppler shift, channel frequency response is calculated by loading the transmitted signal after the fading channel has been applied but before noise is added and dividing it by the clean transmitted signal before the channel is applied. This means that it provides the best estimate of the frequency response at each frequency and on each symbol (BBC R\&D White Paper 196, 2011). As a result of the simulation, it was found that DTG channels based on the Ricean channels had an excellent constellation diagram 
with few constellation points away from the ideal shape. Notice the $75 \mu$ s delays found in this simulation are much larger than those found in (Guerra, 2012). In general, results obtained for all 8 channel models showed that only a slight downward change in the SNR produced a much higher change in BER and most of the error correction was done in LDPC decoder as expected, while BCH decoder corrects only smaller amount of errors. On the other hand, results for the mobile channel models presented many constellation points spread far away from the ideal shape, especially considering higher mobile velocities of about $15 \mathrm{~km} / \mathrm{h}$. In this case, any SNR would produce BER much higher than required for stable reception, which means the possible spectral efficiency introduced through the 256-QAM mode usage still cannot be considered for mobile reception in high speeds. After the simulation, measurements were taken on a receiver which was situated at $11^{\text {th }}$ floor in a high building in urban zone. An antenna with $11 \mathrm{dBi}$ gain, at the relative height of $1.5 \mathrm{~m}$ from the floor was used and the measurement site had a clear view to the transmitter. All measurements were made at the $730 \mathrm{MHz}$ in ultra high frequency (UHF) using a DVB-T2 test signal and the same parameters used in the simulation. Two types of measurements were performed: the first without obstacles (ideal path) and the second with obstacles, resulting in signal attenuations, this attenuation being increased by $1 \mathrm{~dB}$ to the point where decoding of the signal became impossible. The maximum attenuation obtained while decoding was still possible was equal to 10.2 $\mathrm{dB}$ from ideal receiving conditions. Through the results obtained in the two scenarios it is possible to clearly notice the increase of BER when obstacles are considered. These results demonstrate that LDPC of $8.6 .10^{-2}$ BER is very similar to the results obtained in the simulation.

When most of the first-generation DTV standards were constituted in late 90s of the 20 century, an alternative and important technique called beamforming was just an emerging technique at that time, so that those standards did not consider this very promising technology. However, with the rapid development of multi-input and multioutput (MIMO) technology, numerous research and experiments have been carried out for MIMO-based DTV systems to improve the system performance. Based on this a recent approach was considered in (Zhang, 2004). Actually it is proposed that an undesirable interference can be removed perfectly by using a beamformer. Consequently, an improved equalizer performance and additional possibility of increasing spectral efficiency could be achieved in digital terrestrial television (DTT). It is well-known that SFN networks 
often deal with the problem of inter-symbolic interference (ISI) which is mainly caused by two mechanisms - the "natural" dispersion caused by wave components reflected in the vicinity of the receiver, and the artificial delay spread caused by the reception of signals from different sources. The latter is called SFN-structural-multipath as proposed in (Zhang, 2004) and (Guerra, 2012). In traditional digital TV broadcasting system of SFN, the SFN-structural-multipath dispersion are mitigated by means of equalization (P. Angueira et al., 2003), (Ligeti, 1999), (Rebhan, 1993), (Malmgren, 1997). However, if some of the transmitters are located too far away from the receiver, their signals may arrive too late. Instead of contributing to the signal quality these signals turn into interference because of the excessive delay. In this case, the equalizer will fail to work because its observation interval is shorter than the length of the SFN-structural multipath propagation. To deal with a spatial diversity limitation, which is converted into temporal diversity, and a loss of signal space information, a new SFN model was proposed in (Zhang, 2004). In fact, this is a beamforming technique that separates signals transmitted from different directions of arrivals (DOA's) (Kailath, 1980). These signals in turn can be recovered from the multiple output of the beamformer by a filterbank that receives multiple outputs from a block responsible for beamforming. This is indeed a MIMO system (Inouye, 2002), (Tugnait, 2001), (Haykin, 1996) applied to a Single-Carrier (SC) network. The dispersions can be attenuated, since the block responsible for the beamforming in separating the incoming signals filters the structural multipath, while the equalizers with a short observation interval cancel out the inter-symbolic interference caused by the natural dispersions. For the model to behave efficiently it is necessary to know the direction of the desired signal (DOA). In general, since this direction is not known, it is necessary to estimate it, and for that, the estimation method MUSIC (multiple signal classification) is used to find an estimate of a steering vector that maximizes a cost function (Guerra, 2001). In this way, the coefficients of the filters that bring the spatial information of the signals are selected in order to format the radiation pattern reinforcing the reception in certain directions and attenuating in others. This optimization process is obtained by the cost function that contains the steering vector. A computer simulation was conducted to evaluate the performance of the proposed method with that of the traditional equalizer based on the SFN-structural-multipath. Three transmitters were used to send identical signals simultaneously and three channels were constructed to simulate a wireless environment. Notice that in a SFN structure, signals will pass through these channels with different propagation delays and amplitude factors, before arrive at the receiver with 
different incident angles (DOA). These parameters were modified during the experiment so that simulating different environments and channel conditions. Also, to evaluate the performance of the equalizer the mean square error (MSE) was used. The simulation results over three different attempts showed that the traditional equalizer converges at a very slow rate and in the proposed scheme convergence was always faster. So, in the typical SFN environment with heavy ISI, the proposed method based on the new SFN model has a performance advantage over the traditional one found in (Zhang, 2004).

In this final review of the current research development, some relevant parameters related to the multipath behavior of the channel, using field data collected in a real network were considered in (Guerra, 2005). Besides that, the reception conditions associated with a distributed transmission scheme together with an analysis of the influence of the specific environment were also addressed. The measurement occurred through a broadcasting network operating as an SFN in the UHF band. To evaluate the Root Mean Square Delay Spread (RDS) of measured channel, an urban and a semi-urban environment were chosen, in which three transmitters were responsible for covering the measurements of such regions. In order to determine which transmitters operated at each point chosen for testing, the measurements of the delay profile were obtained at points in the region, as defined in (Guerra, 2005), using the impulse response in the transmissions, since previously they already had the theoretical values of the impulse responses of each transmitter. In this way, it was possible to compare and conclude which transmitters were acting at each point. Despite previous knowledge, some points chosen for data acquisition were not clear enough to define how many and which transmitters acted on these points. It is interesting to remember that the RDS parameter evaluated in this article determines the width of the simulated impulse responses, and therefore the propagation time of the echoes present in the channel. It should be noted that all measurements involved were taken at fixed locations and there was no movement involved. The measurements were obtained through an omnidirectional antenna located in the upper part of the used van, totaling around 2.6 meters of the ground, which conforms to the European definitions (Digital Video Broadcasting (DVB), 1997). In (Guerra, 2005) the RDS parameter values reported in section $A$ of table VI were compared with those obtained in (Guerra, 2012) which is listed in Table 1 below. Even in the case of separate campaigns, but involving networks with more than one transmitter, all measurements were made in fixed locations and there was no movement involved. The results of Table 
1 were also calculated using an omni antenna, but at heights of 13.4 meters.

Table 1 - RDS values for three Thresholds

\begin{tabular}{|c|c|c|c|c|}
\hline \multirow{2}{*}{$\begin{array}{c}\text { THRESHOLD } \\
\text { (omni) }\end{array}$} & \multicolumn{2}{|c|}{ Excess delay $(\mu \mathrm{s})$} & \multicolumn{2}{c|}{ RDS $(\mu \mathrm{s})$} \\
\cline { 2 - 5 } & Mean & Max & Mean & Max \\
\hline$-20 \mathrm{~dB}$ & 0.86 & 4.68 & 2.45 & 8.01 \\
\hline$-15 \mathrm{~dB}$ & 1.16 & 4.55 & 2.84 & 7.99 \\
\hline$-10 \mathrm{~dB}$ & 2.45 & 4.48 & 4.37 & 8.04 \\
\hline
\end{tabular}

Despite the divergences precluding a more precise comparison, there are conclusions that can be made. As can be seen, the RDS parameter is much more directly related to reception conditions than to the effects caused by a network with more than one transmitter. Notice that even a slight modification in the height of the measuring antenna is sufficient to produce changes in the parameter in question.

\subsection{Expected Vs. Occurred}

In the near future, analog broadcasting via terrestrial radio distribution systems will be totally replaced by digital systems like ISDB-T and DVB-T which was first designed for stationary and portable reception. However, beyond this portable reception and the modern technical requirement demanded for HDTV, mobile reception services is an increasing interest. Since the expectations obtained in (Guerra, 2012) predicted a possible development of the research focused on the spectral efficiency of digital TV systems, this present work naturally opens up more space for other forecasts and trends related to future technical requirements. A significant destructive spectral interference which could be considered a tremendous limitation in a "standard" Single Frequency Network can be overcome when the DVB project developed the second-generation digital terrestrial television standard, the DVB-T2. In addition, differently from the SISO mode that radiate the same broadcasted signal from two transmitters or more (Guerra, 2012), another important innovation was the possibility of applying the advanced multiple-input single-output (MISO) transmission technique in a SFN. This innovation was immediately translated in lower BER values and consequently the mitigation of the negative impact 
of the Rayleigh channel on the broadcasted HDTV services. In this way, it is not difficult to conceive for a near future the development of new DTV platforms capable of providing high resolution digital TV services for mobile users. Despite of the results obtained in (Tralic, 2012) regarding the 256-QAM mode usage and the necessary stable reception for mobiles in high speeds, the study proposed in (Zhang, 2004) may be one of the solutions to support high data rates in such conditions. Meanwhile, it is known that Japan is studying the MIMO technology for ISDB-T systems and taking into account the use of higher order modulation schemes, such as 1024-QAM, to maximize the system capacity (Linglong, 2012). It is important to notice the performance enhancement attainable with the suggested beamforming technique also depends heavily on the array geometry. In particular, the array geometry should be matched to the spatial characteristics of the radio environment especially considering a high Doppler frequency produced by the velocity of the mobile and the network frequency (Tsoulos, 1997), (Paulraj, 1997). So, for mobile reception at higher moving velocity this should be one way to avoid many constellation points spread far away from the ideal shape of the constellation diagram.

\section{CONCLUSIONS}

In (Guerra, 2012) the measurement campaign carried out in a suburban SFN network with two synchronized transmitters using SISO mode proved an extra transmission capacity, which could be translated in a potential increase of spectral efficiency considering mobile applications in the Brazilian DTV standard. On the other hand, the flexibility and the configuration options provided by the new DVB terrestrial standard (Polak, 2015) confirmed one of our measurements results which is about the possibility of maximizing spectrum efficiency seen today as the right choice for future broadcasting networks and HDTV services. Notice the second-generation digital terrestrial television standard (DVBT2) was developed induced by this very same request which is maximize the spectral efficiency. Also, the negative impact of the Rayleigh channel on the broadcasted HDTV services was highly attenuated with a small increase of BER ratios. This maybe means in a near future the possibility of implementing higher-resolution transmission modes to mobile terminals by adding alternative techniques to the present systems (Zhang, 2004). 
In (Tralic, 2012) measurements of simulated and real channel characteristics in the DVB-T2 system were presented. Considering the performance of the standard in a SFN scenario, it can be concluded that DVB-T2 allows satisfying reception of the signals in various transmission conditions. The MISO mode associated with the DVB-T2 standard is primarily responsible for this performance achieved. Results also show that LDPC decoder performs most of the error corrections resulting in a very robust signal reception and this is automatically reflected for all different types of fading channels considered. Differences in measurement and simulation result are consequence of properties of channel estimator in the receiver. Currently it uses only ideal channel estimation, which means that the channel response is obtained without the pilot signals that requires a SNR $10 \mathrm{~dB}$ higher. In comparison with the SISO mode presented in (Guerra, 2012) where significant destructive spectral interference is translated in higher BER values, the DVBT2 MISO principle has been the principal contribution associated with the actual DTV platform that fulfill modern technical requirements and meet user and market demands for HDTV services.

The method proposed in (Zhang, 2004) outperforms the traditional equalizer which is based on the SFN-structural-multipath. It is necessary to stress that, despite being a solution applied to simulated channels, this new solution can be extended to real conditions where channel dispersion parameters are priorities. Additionally, it is also important to notice that by using the beamforming technique the structural multipath can be removed so that releasing "space" in the guard interval. This, in turn, directly implies in a possibility of increasing the spectral efficiency of the system, as suggested in (Guerra, 2012).

Lastly, the article presented in (Guerra, 2005) regards a SFN network that gives coverage to the urban area of the city of Madrid and its surroundings. The study involved the relationship between the environment type and the SFN effect. Actually, the obtained results do not allow to associate high RDS values to the SFN effect and an increase in the RDS is more associated to the reception conditions. In other words, from table I we could confirm that a simple variation in antenna height, which also represents a small variation of the reception conditions were sufficient to produce significantly higher RDS values. I conclude that the way is to develop more technologies capable of efficiently mitigate the degradation generated by environmental conditions. 


\section{REFERENCES}

Angueira, P. DTV (COFDM) SFN signal variation field tests in urban environments for portable outdoor reception. IEEE Trans. Broadcast, vol. 49, pp. 81-86, 2003.

BBC R\&D White Paper 196. The Common Simulation Platform. Disponível em <http:// downloads.bbc.co.uk/rd/pubs/whp/whp-pdffiles/WHP196.pdf> Acesso em: Jan. 2011.

Digital Video Broadcasting (DVB). Implementation Guidelines for DVB Terrestrial Services. European Telecommunications Standards Institute, 1997.

DVB BlueBook A133. Implementation guidelines for a second generation digital terrestrial television broadcasting system. DVB Document, February, 2009.

DVB Document A122. Frame structure channel coding and modulation for a second generation digital terrestrial television broadcasting system. June, 2008.

DVB Fact Sheet: DVB. 2nd Generation Terrestrial Broadcasting. DVB Project Office, April 2012

Eizmendi, I., Vélez, M. CNR requirements for DVB-T2 fixed reception based on field trial results. Electronics Letters, vol. 47, pp. 57-59, 2011.

ETSI EN 300744 (V1.6.1). Framing structure, channel coding and modulation for digital terrestrial television. September, 2008.

Fisher, W. Digital Video and Audio Broadcasting Technology. A Practical Engineering Guide. Springer, 2010 
Guerra, G., Angueira, P., Vélez, M. Field Measurement Based Characterization of the Wideband Urban Multipath Channel for Portable DTV Reception in Single Frequency Networks. IEEE Transactions on Broadcasting, vol. 51, no. 2, 2005.

Guerra, M. Experimental Characterization of a SFN Digital Broadcast Channel. IEEE Latin-America Conf. on Commun, pp. 1-4, 2012.

Guerra, M. Utilização de Antenas Adaptativas em Sistemas CDMA. Rio de Janeiro: Center for Telecommunication Studies, Catholic University of Rio de Janeiro, Rio de Janeiro, 2001.

Haykin, S. Adaptive Filter Theory. Prentice Hall, 1996

Inouye, Y., Liu, R. W. A system-theoretic foundation for blind equalization of an FIR MIMO channel system. IEEE Trans., vol. 49, no. 4, 2002.

Kailath, T. Linear System. Prentice Hall, 1980.

L. Eizmendi, L. Empirical DVB-T2 Thresholds for Fixed Reception. IEEE Transactions on Broadcasting, vol. 59, pp. 306-316, 2013.

Ligeti, A. Minimal cost coverage planning for single frequency networks. IEEE Trans. Broadcast, vol. 45, pp. 78-87, 1999.

Linglong, D., Zhaocheng, W., Zhixing, Y. Next-Generation Digital Television Terrestrial Broadcasting systems: Key Technologies and Research Trend. IEEE Communications Magazine, pp. 150-158, 2012.

Malmgren, G. On the performance of single frequency networks in correlated shadow fading. IEEE Trans. Broadcast, vol. 43, no. 2, 1997. 
Morgade, J. A measurement-based methodology for the DVB-T2 MISO/SISO gain characterization in experimental networks. 15th Int. Conf. on Electromagnetics in Advanced Applications, pp. 487-490, 2013.

Morgade, J. SFN-SISO and SFN-MISO Gain Performance Analysis for DVB-T2 Network Planning. IEEE Transactions on Broadcasting, vol. 60, pp. 271-286, 2014.

Paulraj, A., Papadias, B. Space-Time Processing for Wireless Communications. IEEE Personal Communications, vol. 14, pp. 49-83, 1997.

Polak, L., Kratochvil, T. DVB-T and DVB-T2 Performance in Fixed Terrestrial TV Channels. Int. Conf. Telecommunication and Signal Processing, pp. 725-729, 2012.

Polak, L., Kratochvil, T. SISO/MISO Performances in DVB-T2 and Fixed TV Channels. Int. Conf. Telecommunication and Signal Processing, pp.768-771, 2015.

Rebhan, R. On the outage probability in single frequency networks for digital broadcasting. IEEE Trans. Broadcast, vol. 39, pp. 395-401, 1993.

Richardson, T., Urbanke, R. The Renaissance of Gallager's Low-Density Parity-Check Codes. IEEE Commun. Magazine, vol. 41, pp.126-131, 2003.

Rohde \& Schwarz. Generating a Test Signal for Distributed DVB-T2 MISO. Application Note 7BM80_1E, 2012.

Takada, M. Transmission system for ISDB-T. Proceeding of the IEEE, vol. 94, pp. 251256, 2006.

Tralic, D., Dumic, E., Vukovic, J., Grgic, S. Simulation and Measurement of DVB-T2 Channel Characteristics. In ELMAR-2012, Croatia, September, 2012. 
TS 102831 (V1.2.1). Implementation guidelines for a second generation digital terrestrial television broadcasting system. August, 2012.

Tsoulos, G., Beach, M., Geehan, J. Wireless Personal Communications for the 21st Century:European Technological Advances in Adaptive Antennas. IEEE Communications Magazine, vol. 35, pp. 102-109, 1997.

Tugnait, K., Huang, B. Blind estimation and equalization of MIMO channels via multidelay whitening. IEEE J. Sel. Areas Commun., vol. 19, no. 8, 2001.

Zhang, L., Gui, L., Qiao, Y., Zhang, W. Obtaining Diversity Gain for DTV by Using MIMO Structure in SFN. IEEE Transaction on Broadcasting, vol. 50, 2004. 